\title{
Mass of ${ }^{36} \mathrm{Ca} \dagger$ \\ R. E. Tribble, * J. D. Cossairt, and R. A. Kenefick \\ Cyclotron Institute and Physics Department, Texas A\&M University, College Station, Texas 77843
}

(Received 14 October 1976)

\begin{abstract}
The ${ }^{40} \mathrm{Ca}\left({ }^{4} \mathrm{He},{ }^{8} \mathrm{He}\right){ }^{36} \mathrm{Ca}$ reaction has been used to provide the first observation of the nuclide ${ }^{36} \mathrm{Ca}$. The $Q$ value and mass excess were found to be $-57.58 \pm 0.04$ and $-6.44 \pm 0.04 \mathrm{MeV}$, respectively. The new mass completes four members of the $A=36$ isobaric quintet and hence serves as a test of the isobaric multiple mass equation.
\end{abstract}

$$
\left[\begin{array}{c}
\text { NUCLEAR REACTIONS }{ }^{40} \mathrm{Ca}\left({ }^{4} \mathrm{He},{ }^{8} \mathrm{He}\right) . \text { Measured }{ }^{36} \mathrm{Ca} \text { mass. Deduced coeffi- } \\
\text { cients of the isobaric multiplet mass equation. }
\end{array}\right]
$$

\section{INTRODUCTION}

In this paper we report the first observation of the $T_{z}=-2$ nuclide ${ }^{36} \mathrm{Ca}$. The mass measurement result completes the fourth member of the $A=36$ isobaric quintet and thus provides a test of the isobaric multiplet mass equation (IMME).

The quadratic IMME predicts that the masses of isobaric multiplets will be related by the equation $M\left(A, T, T_{\varepsilon}\right)=a(A, T)+b(A, T) T_{z}+c(A, T) T_{z}{ }^{2}$, where $a, b$, and $c$ are constants for a multiplet of isospin $T{ }^{1}$ The IMME has been tested extensively with isobaric quartets. Only in $A=9$ has any significant deviation been determined. With the IMME parametrized to higher order in $T_{z}$ as $d T_{z}{ }^{3}$, the most recent $A=9$ isobaric quartet results give $d=\mathbf{5 . 8}$ $\pm 1.5 \mathrm{keV} .^{2}$ The masses of two other $T_{\varepsilon}=-2 \mathrm{nu}-$ clei, ${ }^{8} \mathrm{C}$ (Ref. 3) and ${ }^{20} \mathrm{Mg}$ (Ref. 4) completed either four $(A=20)$ or five $(A=8)$ isobaric quintet members. The previous isospin quintet results sug gested possible, albeit small, deviations from the quadratic IMME. Further isospin quintet tests could help discern any systematic deviations. The result for the $A=36$ quintet provides an additional IMME test, and further, $A=36$ represents the highest $Z$ mass quintet that is presently feasible to complete.

\section{EXPERIMENTAL PROCEDURE AND RESULTS}

The mass of ${ }^{36} \mathrm{Ca}$ was determined by the fourneutron pickup reaction ${ }^{40} \mathrm{Ca}\left({ }^{4} \mathrm{He},{ }^{8} \mathrm{He}\right){ }^{36} \mathrm{Ca}$, with a 121.4-MeV $\alpha$ beam from the Texas A\& M University 88 -inch cyclotron, incident on a $3-\mathrm{mg} /$ $\mathrm{cm}^{2}$ natural $\mathrm{Ca}$ target. The reaction products were detected in the focal plane of an Enge splitpole magnetic spectrograph by a $10-\mathrm{cm}$ singlewire gas proportional counter which was backed by a $50-\mathrm{mm} \times 10-\mathrm{mm} \times 600-\mu \mathrm{m}$ Si solid-state detector. Particle position was determined by charge division and particle identification was constrained according to the three parameters
(1) $(d E / d x)_{\text {gas }}$, (2) $E$, and (3) time of flight (TOF) relative to the cyclotron $\mathrm{rf}$. Pileup rejection was used with the solid-state detector in order to prevent any degradation in resolution due to high count rates. A $0.127-\mathrm{mm}$ Kapton absorber foil was inserted between the gas proportional counter and the solid-state detector so that ${ }^{8} \mathrm{He}$ particles would stop in the Si detector. The resulting ${ }^{8} \mathrm{He}$ energy group is easily distinguished from other species as indicated by a plot of $E$ vs TOF, shown in Fig. 1. The superior energy signal obtained from the solid-state detector reduced the accidental background to a level somewhat less than $0.5 \mathrm{nb} / \mathrm{sr} \mathrm{MeV}$.

The incident $\alpha$ beam energy was determined via the momentum matching technique by observation of proton elastic scattering from ${ }^{28} \mathrm{Si}$ simultaneously with deuterons from the ${ }^{28} \mathrm{Si}(p, d)^{27} \mathrm{Si}$ (g.s.) reaction, at a spectrog raph angle of $\theta_{1 \mathrm{ab}}=20^{\circ}$. Protons were obtained from an $\mathrm{H}_{2}^{+}$beam that was

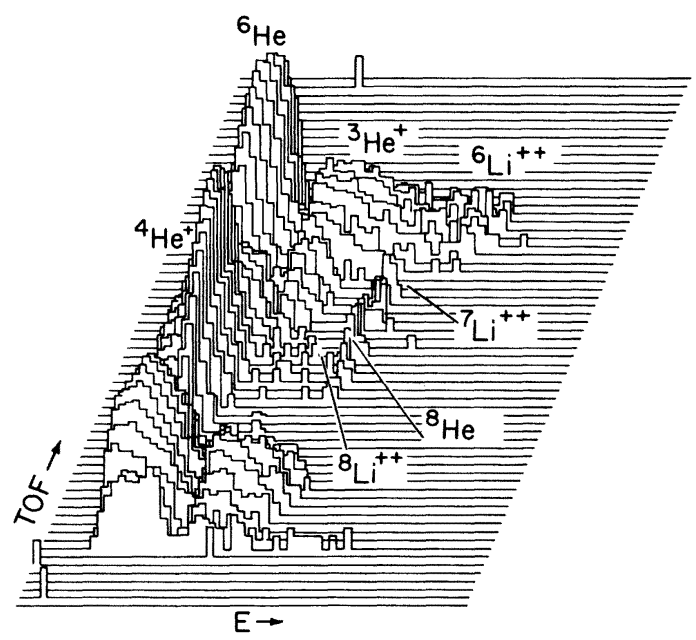

FIG. 1. A spectrum of $E$ vs TOF with the $Z$ dimension (number of counts) logarithmic. Several particle groups are indicated. 


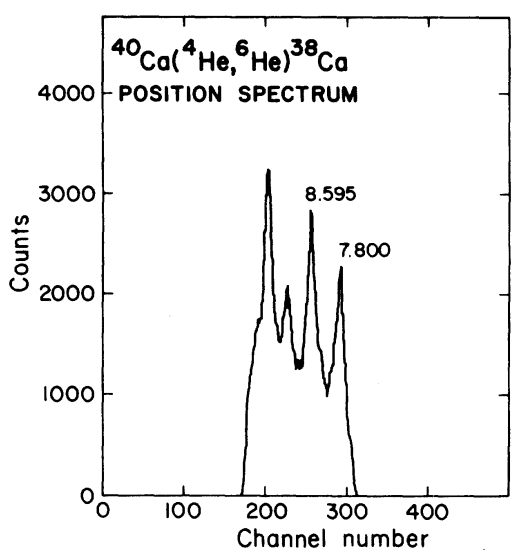

FIG. 2. ${ }^{6} \mathrm{He}$ spectrum populating states in ${ }^{38} \mathrm{Ca}$. Excitation energies for the two states that were used as a calibration check are shown.

accelerated to the same magnetic rigidity as the incident $\alpha$ beam. Previously the spectrograph scattering angle had been measured optically to a precision of $<0.1^{\circ}$ for the angular region that encompassed both the energy calibration and the mass measurement.

The spectrograph focal plane calibration was determined from elastic scattering of $\alpha$ particles on ${ }^{40} \mathrm{Ca}$ in the same configuration that was used for the mass measurement, except for slightly lower magnetic field and reduced entrance aperture. At the ${ }^{8} \mathrm{He}$ magnetic field, a position spectrum from ${ }^{40} \mathrm{Ca}\left({ }^{4} \mathrm{He},{ }^{6} \mathrm{He}\right){ }^{38} \mathrm{Ca}$, shown in Fig. 2, was obtained simultaneously with the ${ }^{8} \mathrm{He}$ spectrum. The ${ }^{6} \mathrm{He}$ events spanned an excitation region from $\sim 7.5$ to $\sim 10 \mathrm{MeV}$ in ${ }^{38} \mathrm{Ca}$. Two states have been observed in this excitation region via ${ }^{40} \mathrm{Ca}(p, t){ }^{38} \mathrm{Ca}$ (Ref. 5) and thus they provided a check of the focal plane calibration and the ${ }^{40} \mathrm{Ca}$ target thickness.

The target thickness was obtained from an ${ }^{241} \mathrm{Am}$

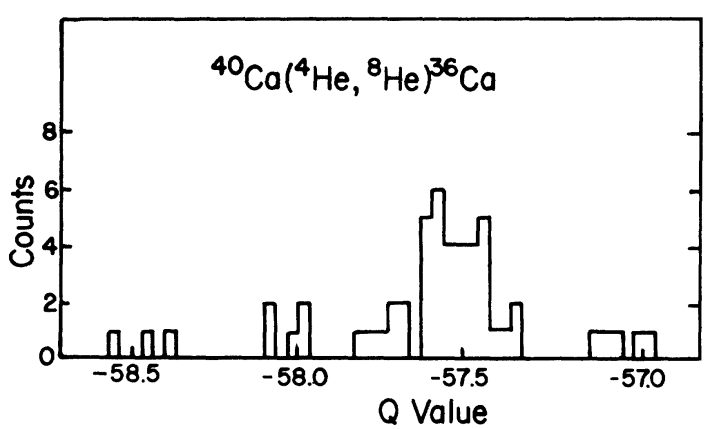

FIG. 3. Spectrum of ${ }^{8} \mathrm{He}$ events populating the ground state of ${ }^{36} \mathrm{Ca}$.

$\alpha$ energy loss measurement. Previous experience has indicated that this method is accurate to $\sim 10 \%$ for foil thicknesses that can be checked by weighing. As was mentioned above, the ${ }^{6} \mathrm{He}$ peaks provided an additional consistency check on the target thickness measurement.

The ${ }^{8} \mathrm{He}$ position spectrum, taken at the laboratory scattering angle $\theta_{1 \mathrm{ab}}=5^{\circ}$ is shown in Fig. 3 . The peak, which is assumed to be the ground state excitation of ${ }^{36} \mathrm{Ca}$, represents a laboratory cross section of $1.3 \pm 0.6 \mathrm{nb} / \mathrm{sr}$ averaged over the $2.3-$ msr spectrograph solid angle. The centroid uncertainty, assuming a background contribution, is $30 \mathrm{keV}$. Combining this with the uncertainties associated with (1) beam ene rgy (10 keV), (2) scattering angle (5 keV), (3) focal plane calibra tion $(15 \mathrm{keV})$, target thickness $(20 \mathrm{keV})$, along with the ${ }^{8} \mathrm{He}$ mass excess of $31.601 \pm 0.013 \mathrm{MeV},{ }^{6}$ we find the reaction $Q$ value to be $-57.58 \pm 0.04 \mathrm{MeV}$, and the mass of ${ }^{36} \mathrm{Ca}$ to be $-6.44 \pm 0.04 \mathrm{MeV}$.

\section{DISCUSSION}

The mass of ${ }^{36} \mathrm{Ca}$ constitutes the fourth measured mass of the $A=36$ quintet. The properties

TABLE I. Properties of the $A=36$ isobaric quintet members and the IMME coefficients.

\begin{tabular}{|c|c|c|c|c|c|}
\hline Nuclide & $T_{\varepsilon}$ & $\begin{array}{c}\text { Mass excess } \\
(\mathrm{keV})\end{array}$ & $\begin{array}{c}E_{x} \\
(\mathrm{keV})\end{array}$ & & Ref. \\
\hline${ }^{36} \mathrm{Ca}$ & -2 & $-6440 \pm 40$ & 0.0 & & Present work \\
\hline${ }^{36} \mathrm{Ar}$ & 0 & $-19376.3 \pm 1.6$ & $10854.2 \pm 1.6$ & & 10 \\
\hline${ }^{36} \mathrm{Cl}$ & 1 & $-25222.3 \pm 1.1$ & $4299.5 \pm 1.1$ & & 11 \\
\hline${ }^{36} \mathrm{~S}$ & 2 & $-30666.0 \pm 1.5$ & 0.0 & & 12 \\
\hline \multicolumn{6}{|c|}{ Predicted coefficients (in keV) for the IMME } \\
\hline$a$ & & $b$ & $c$ & $d$ & $\chi^{2}$ \\
\hline$-19376.0 \pm 1.6$ & & $-6048.1 \pm 3.2$ & $201.6 \pm 1.5$ & $\cdots$ & 0.78 \\
\hline$-19376.3 \pm 1.6$ & & $-6050.3 \pm 4.0$ & $205.8 \pm 5.0$ & $-1.6 \pm 1.8$ & $\cdots$ \\
\hline
\end{tabular}


TABLE II. Coulomb energies for the $A=36$ isobaric quintet. The results are in good agreement with the calculations if the charge-dependent contributions are included. The uncertainties for the experimental results given in parentheses are in $\mathrm{keV}$.

\begin{tabular}{cccc}
\hline \hline & $\begin{array}{c}\text { Calculated Coulomb } \\
\text { contribution } \\
\text { Nuclide }\end{array}$ & $\begin{array}{c}\text { Including charge } \\
\text { dependence } \\
(\mathrm{keV})\end{array}$ & $\begin{array}{c}\text { Experimental } \\
\Delta E_{c}\end{array}$ \\
\hline${ }^{36} \mathrm{Cl}$ & 6368 & 6210 & $6224(2)$ \\
${ }^{36} \mathrm{Ar}$ & 6681 & 6628 & $6629(2)$ \\
${ }^{36} \mathrm{~K}$ & 6994 & 7047 & $7033(6)^{\mathrm{a}}$ \\
${ }^{36} \mathrm{Ca}$ & 7307 & 7465 & $7469(40)^{\mathrm{a}}$ \\
\hline \hline
\end{tabular}

${ }^{\mathrm{a}}$ Based on the quadratic IMME prediction for the ${ }^{36} \mathrm{~K} T=2$ state of $-13126.3 \pm 5.0 \mathrm{keV}$.

of the known multiplet members are given in Table I. We note that all of these multiplet members are particle stable with respect to isospin allowed particle decays and hence no energy shifts due to unbound levels are expected. The quintet members are in relatively good accord with the quadratic IMME as evidenced by a $\chi^{2}=0.78$. Using a cubic IMME we find a predicted $d$ coefficient of $d=-1.6 \pm 1.8 \mathrm{keV}$ which represents a small deviation that is consistent with zero. The coefficients for both the quadratic and quartic fits are given in Table I.

The three isobaric mass quintets $A=8,20$, and 36 have four or more members known and in each case the $T_{z}=-2$ member has been observed via the $\left({ }^{4} \mathrm{He},{ }^{8} \mathrm{He}\right)$ reaction. Thus it is now possible to discuss both the IMME quintet systematics and the cross section dependence of the $\left({ }^{4} \mathrm{He},{ }^{8} \mathrm{He}\right)$ reaction. In the case of $A=8$, all five multiplet masses are known and both $d$ and $e\left(T_{z}^{4}\right)$ coefficients can be deduced. The most recent results give $d=4.4$ $\pm 3.0 \mathrm{keV}$ and $e=2.4 \pm 2.2 \mathrm{keV}$ (Ref. 7). In $A=20$ four multiplet members are known and in this case the deduced $d$ coefficient is $d=-2.3 \pm 2.0 \mathrm{keV}$ (Ref. 4). Deviations from the quadratic IMME appear to be quite small, even in the $A=8$ case which is expected to be the best candidate for a deviation. These results are similar to the excellent description of isospin quartets obtained by the quadratic IMME. Thus it appears unlikely that any isospin quintets will have sizable deviations from the quadratic IMME. We should note, however, that isospin mixing in the $T_{z}=0$ configuration could cause deviations from the quadratic IMME for isospin quintets but not quartets. Such mixing would be expected to give rise to a small $e$ coeffi cient (less than a few keV), and hence will be difficult to determine.

The laboratory cross sections for $\left({ }^{4} \mathrm{He},{ }^{8} \mathrm{He}\right) \mathrm{re}-$ actions populating $T_{z}=-2$ nuclei at $\theta_{1 \mathrm{ab}}=5^{\circ}$ and
$E_{\alpha} \approx 120 \mathrm{meV}$ are (1) $9 \pm_{3}^{2} \mathrm{nb} / \mathrm{sr}-{ }^{8} \mathrm{C}$ (Ref. 3), (2) $3 \pm 1.5 \mathrm{nb} / \mathrm{sr}-{ }^{20} \mathrm{Mg}$ (Ref. 4), and the present result, (3) $1.3 \pm 0.6 \mathrm{nb} / \mathrm{sr}-{ }^{36} \mathrm{Ca}$. The cross section systematics indicate an $\sim 1 / A$ falloff up to ${ }^{40} \mathrm{Ca}$. These results also indicate that the $\left({ }^{4} \mathrm{He},{ }^{8} \mathrm{He}\right)$ cross section to other $T_{z}=-2$ nuclei would not be expected to be more than a few $\mathrm{nb} / \mathrm{sr}$ at forward angles. Very little information is available on the angular dependence of the cross sections. How ever for $A=8$, and $A=20$, experiments at $E=156$ $\mathrm{MeV}$ and $\theta_{1 \mathrm{ab}}=2^{\circ}$ gave cross sections of $\sim 20 \mathrm{nb} / \mathrm{sr}$ for ${ }^{8} \mathrm{C}$ and $\sim 7 \mathrm{nb} / \mathrm{sr}$ for ${ }^{20} \mathrm{Mg}$ (Ref. 8). These re sults indicate a fairly strong forward peaking which is in agreement with predictions from cluster model calculations.

Since the deviations from the quadratic IMME in $A=36$ appear to be small, the mass of the ${ }^{36} \mathrm{~K}$ $(T=2)$ state can now be predicted. Then the Coulomb energies for the $T=2$ states in $A=36$ can be extracted and compared with the calculation of Sherr and Talmi which includes a possible nuclear charge-dependent contribution to the Coulomb energies for $\left(d_{3 / 2}\right)^{n}$ configurations. ${ }^{9}$ In Table II we give the predictions as obtained from Ref. 9 along with the quintet results assuming the IMME result for the ${ }^{36} \mathrm{~K}$ mass excess. The agreement is quite good for the Coulomb energies that include the charge-dependent contributions. As was pointed out by Sherr and Talmi, the charge-dependent interaction that they have calculated would not give rise to a significant deviation from the quadratic IMME but rather would renormalize the coefficients compared with the purely electromagnetic result. The excellent agreement of the corrected Coulomb energies with available experimental data is quite suggestive of the need for a small charge-dependent nuclear interaction. However, a measurement of the ${ }^{36} \mathrm{~K}(T=2)$ mass would stringently test both the IMME and these Coulomb energy predictions. 
$\dagger$ Supported in part by the National Science Foundation. *Alfred P. Sloan Fellow.

${ }^{1}$ E. P. Wigner, in Proceedings of the Robert A. Welch Foundation Conference on Chemical Research, Houston, Texas, edited by W. O. Milligan (Robert A. Welch Foundation, Houston, Texas, 1957), p. 67.

${ }^{2}$ E. Kashy, W. Benenson, D. Mueller, R. G. H. Robertson, and D. R. Goosman, Phys. Rev. C 11, 1959 (1975).

${ }^{3}$ R. E. Tribble, R. A. Kenefick, and R. L. Spross, Phys. Rev. C 1, 50 (1976).

${ }^{4}$ R. E. Tribble, J. D. Cossairt, and R. A. Kenefick, Phys. Lett. 61B, 353 (1976).

${ }^{5}$ R. A. Paddock, Phys. Rev. C 5, 485 (1972).

${ }^{6}$ The ${ }^{8} \mathrm{He}$ mass excess is the average of three determinations (all in MeV): $31.611 \pm 0.017$, R. Kouzes and W. H. Moore, Phys. Rev. C 12, 1511 (1975); 31.600 \pm 0.025 , J. W. Jänecke, F. D. Becchetti, L. Chua, and A. VanderMolen, ibid. 11, 2114 (1975); $31.57 \pm 0.03$,
J. Cerny, N. A. Jelley, D. L. Hendrie, C. F. Maguire, J. Mahoney, D. K. Scott, and R. B. Weisenmiller, ibid. 10, 2654 (1974).

${ }^{7}$ R. G. H. Robertson, W. Benenson, E. Kashy, and D. Mueller, Phys. Rev. C 13, 1018 (1976).

${ }^{8}$ R. G. H. Robertson, S. Martin, W. R. Falk, D. Ingham, and A. Djaloeis, Phys. Rev. Lett. 32, 1207 (1974).

${ }^{9}$ R. Sherr and I. Talmi, Phys. Lett. $\overline{56}$ B, 212 (1975).

${ }^{10}$ A. Huck, G. J. Costa, G. Walter, M. M. Aleonard, J. Dalmas, P. Hiebert, F. Leccia, P. Mennrath, J. Vernotte, M. Langevin, and J. M. Maison, Phys. Rev. C 13, 1786 (1976).

${ }^{11} \mathrm{~J}$. Vernotte, S. Fortier, M. Langevin, J. M. Maison, M. Vergnes, and B. H. Wildenthal, Phys. Rev. C 13 , 461 (1976).

${ }^{12}$ P. M. Endt and C. van der Leun, Nucl. Phys. A214, 1 (1973). 